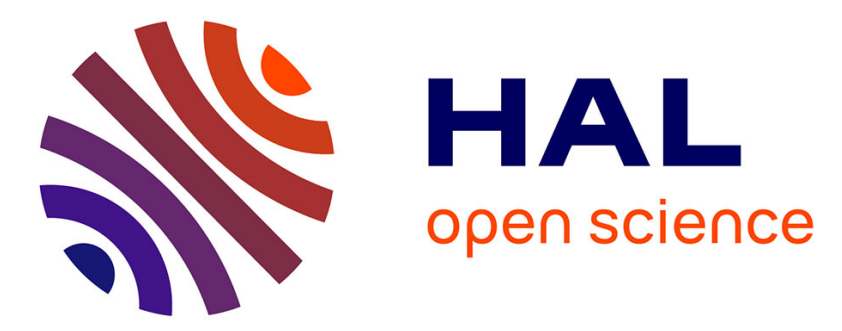

\title{
Shuffling for understanding multifractality, application to asset price time series
}

Patrice Abry, Yannick Malevergne, Herwig Wendt, Marc Senneret, Laurent Jaffrès, Blaise Liaustrat

\section{- To cite this version:}

Patrice Abry, Yannick Malevergne, Herwig Wendt, Marc Senneret, Laurent Jaffrès, et al.. Shuffling for understanding multifractality, application to asset price time series. European Signal Processing Conference (EUSIPCO), Sep 2019, A Coruna, Spain. hal-02361738

\section{HAL Id: hal-02361738 \\ https://hal.science/hal-02361738}

Submitted on 13 Nov 2019

HAL is a multi-disciplinary open access archive for the deposit and dissemination of scientific research documents, whether they are published or not. The documents may come from teaching and research institutions in France or abroad, or from public or private research centers.
L'archive ouverte pluridisciplinaire HAL, est destinée au dépôt et à la diffusion de documents scientifiques de niveau recherche, publiés ou non, émanant des établissements d'enseignement et de recherche français ou étrangers, des laboratoires publics ou privés. 


\section{Shuffling for understanding multifractality, application to asset price time series}

\author{
Patrice Abry \\ Université de Lyon, ENS de Lyon \\ CNRS, Laboratoire de Physique \\ Lyon, France \\ patrice.abry@ens-lyon.fr
}

\author{
Yannick Malevergne \\ Université Paris 1 Panthéon-Sorbonne \\ PRISM Sorbonne and Labex Refi \\ Paris, France \\ yannick.malevergne@univ-paris1.fr
}

\author{
Herwig Wendt \\ Université de Toulouse \\ CNRS, IRIT \\ Toulouse, France \\ herwig.wendt@irit.fr
}

\author{
Marc Senneret \\ Vivienne Investissement \\ Lyon, France \\ msenneret@vivienne-im.com
}

\author{
Laurent Jaffrès \\ Vivienne Investissement \\ Lyon, France \\ ljaffres@vivienne-im.com
}

\author{
Blaise Liaustrat \\ Vivienne Investissement \\ Lyon, France \\ blaustriat@vivienne-im.com
}

\begin{abstract}
Multifractal analysis has become a standard signal processing tool successfully used to model scale-free temporal dynamics in many applications, very different in nature. This is notably the case in financial engineering where, after Mandelbrot's seminal contributions, multifractal models have been used since the late 90ies to describe temporal fluctuations in asset prices. However, what exact features of temporal dynamics are actually encoded in multifractal properties remains generally only partially understood. In finance, notably, multifractality is associated to the burstiness of the returns, yet its relation to trends (signs of the returns) or volatility (modulus of the returns) remains unclear. Comparing the estimated multifractal properties of well-controlled synthetic multifractal processes to those of surrogate data, obtained by applying random permutations (shuffling) either to signs, or to modulus, or to both, of increments of original data, permits to better understand what aspects of temporal dynamics are captured by multifractality. The same procedure applied to a large dataset of asset prices entering the composition of the Eurostoxx600 index permits to evidence a simple and solid relation between multifractality and volatility as well as a weaker and complicated relation to returns.
\end{abstract}

Index Terms-Multifractality, scale-free temporal dynamics, asset prices, finance, shuffling, surrogate.

\section{INTRODUCTION}

Context. Self-similarity has been massively used in many applications very different in nature to model scale-free temporal dynamics, that is, temporal dynamics that are not governed by any preferred time scale, in signals (cf. e.g., [1]). Selfsimilarity (or its asymptotic counterpart, long range dependence (LRD)) essentially models a slow power law decay of the covariance function at large lags [13], thus indicating that correlations amongst samples of data remain significant even for samples very far apart [1]. LRD is quantified by departures of the Hurst parameter $H$ from 1/2. Multifractality complements and enriches LRD by introducing versatility in the modeling of scale-free dynamics at all statistical orders. It accounts for potentially different scaling exponents, when self-similarity actually implies that scale-free dynamics at any finite statistical order are controlled uniquely by $H$. In finance, it is well documented that asset prices show no temporal correlation $(H=0.5$, the efficient market hypothesis (EMH)) but have some structured scale-free dependencies, sometimes quantified by the fact that volatility (squared returns) show LRD with $H>0.5$, which prompted for the use of multifractality to model asset price fluctuations [6], [8].

However, what aspects of temporal dynamics are actually encoded by multifractality remains not very well understood. Despite being crucial for a better understanding of the underlying mechanisms governing temporal dynamics, this open question has rarely been studied theoretically and barely investigated on real-world data. For instance, in finance, multifractality has been related to the stage of development of financial markets and EMH [8], [16] or to the way volatility relaxes after endogenous or exogeneous shocks [4], [14] but it remains an open question to assess whether multifractality encodes the temporal fluctuations in return signs or in volatility.

Related work. At the theoretical level, it was proven that, for fractional Brownian motion, the reference process for modeling scale-free dynamics, LRD is associated with the fluctuations of the signs of its increments, while their modulus only shows short-term dependencies [13]. To the best of our knowledge, similar theoretical results do not exist for multifractal processes and the question remains barely studied empirically.

In a seminal contribution [12], Mandelbrot introduced multifractality in the modeling of asset price time series to account for burstiness, departures from Gaussianity, large size fluctuations and scale-free dynamics. This paved the way to numerous studies consistently reporting multifractal dynamics in financial time series [1], [3], [5], [10]. It renewed to some extent financial engineering, notably by suggesting to replace the Markov Switching Model used to describe the fluctuations of volatility, which is by construction based on the existence of a few typical time scales to be identified, by a multifractal switching model [9]. However, the understanding of what mechanisms in pricing are actually encoded in multifractality 
remains an open question and an obvious preliminary step for actual use in financial engineering.

Goals, contributions and outline. Making use of random permutation (or shuffling) procedures, that consist in destroying a targeted property of data while preserving others unaltered, the present work aims to provide a better understanding of whether multifractality encodes the temporal dynamics of the signs or of the modulus in a multifractal time series, both at a general level and in the precise context of asset price analysis. To that end, Section II restates the key notions for theoretical and practical multifractal analysis, recalls the paradigm model of the fractional Brownian motion in multifractal time and defines the shuffling procedures used here. Section III reports Monte Carlo simulations comparing the multifractal properties estimated from a large number of independent copies of fractional Brownian motion in multifractal time to those estimated from surrogate versions of such data, obtained from the application of several different shuffling procedures. This permits to evidence relations between, on one hand, LRD and the temporal dynamics of signs, and on other hand, multifractality and the temporal dynamics of the modulus. Section IV compares the multifractal properties of a large dataset of financial times series consisting of daily prices for 244 assets contributing to the Eurostoxx600 index. Result shows that multifractality is strongly associated with the temporal dynamics of volatility.

\section{Multifractal ANALYSis AND SHUFFLING}

\section{A. Multifractal analysis: Theory}

Local regularity. Multifractal analysis aims at characterizing the fluctuations along time of the local regularity of a signal $X(t)$, cf., e.g., [17]. Local regularity can be quantified by pointwise exponents, the most commonly used being the Hölder exponent, $h(t)>0$, defined as follows: $X$ is said to be in $C^{\alpha}(t), \alpha \geq 0$, if there exist a polynomial $P_{t}$ with $\operatorname{deg}\left(P_{t}\right)<\alpha$ and a constant $C>0$ such that: $\mid X(t+a)-P_{t}(t+$ $a)\left.|\leq C| a\right|^{\alpha}$ when $|a| \rightarrow 0$. The Hölder exponent consists of the largest such $\alpha: h(t) \triangleq \sup \left\{\alpha: X \in C^{\alpha}(t)\right\} \geq 0$. In essence, the larger $h(t)$, the smoother $X$ around $t$, and conversely, the closer $h(t)$ to 0 , the more irregular $X$ at $t$.

Multifractal spectrum. Despite being based on a local measure of regularity, multifractal theory does not, however, base analysis on the time evolution of the function $h(t)$ itself, but instead aims to provide a global and geometrical description of the temporal dynamics of $X$ with the so-called multifractal spectrum $\mathcal{D}(h)$. It is defined as the collection of Hausdorff dimensions $\operatorname{dim}_{H}$ of the sets of points $t \in \mathbb{R}$ where $h(t)$ takes the same value $h$ [17]: $\mathcal{D}(h) \triangleq \operatorname{dim}_{H}(t: h(t)=h)$. The multifractal spectrum $\mathcal{D}(h)$ can thus be considered as an efficient summary for the temporal dynamics of $X$.

\section{B. Multifractal analysis: formalism}

The practical estimation procedure for $\mathcal{D}(h)$ from data is referred to as the mutlifractal formalism, and requires the use of multiscale quantities, that match the pointwise exponent chosen to quantify regularity. It is well documented that measuring the Hölder exponent calls for the use of wavelet leaders defined as follows [17]. Let $d_{X}(j, k)=2^{-j / 2}\left\langle\psi_{j, k} \mid X\right\rangle$ denote the discrete wavelet transform (DWT) coefficients of $X$, with $\psi$ the mother wavelet, an oscillating reference pattern, characterized by its number of vanishing moments $N_{\psi}$, a positive integer defined as $\psi \in C^{N_{\psi}-1}$ and $\forall n=0, \ldots, N_{\psi}-1$, $\int_{\mathbb{R}} t^{k} \psi(t) d t \equiv 0$ and $\int_{\mathbb{R}} t^{N_{\psi}} \psi(t) d t \neq 0$. Further, $\psi$ is chosen such that the collection of dilated and translated templates $\left\{\psi_{j, k}(t)=2^{-j / 2} \psi\left(2^{-j} t-k\right)\right\}_{(j, k) \in \mathbb{Z}^{2}}$ forms an orthonormal basis of $\mathcal{L}^{2}(\mathbb{R})[11]$.

Wavelet leaders are defined as local suprema of wavelet coefficients: $\ell_{X}(j, k) \triangleq \sup _{2^{j^{\prime}} k^{\prime} \in 3 \lambda_{j, k}}\left|d_{X}\left(j^{\prime}, k^{\prime}\right)\right|$, with $\lambda_{j, k}=$ $\left[k 2^{j},(k+1) 2^{j}\right)$ the dyadic interval of size $2^{j}$ and $3 \lambda_{j, k} \triangleq$ $\lambda_{j, k-1} \cup \lambda_{j, k} \cup \lambda_{j, k+1}$ the union of $\lambda_{j, k}$ with its 2 neighbors. It can be shown that wavelet leaders reproduce Hölder exponents in the limit of fine scales, $L_{X}(j, k) \sim C 2^{j h(t)}$ as $2^{j} \rightarrow 0$ for $t=2^{j} k$ and hence that [17]:

$$
\frac{1}{n_{j}} \sum_{k=1}^{n_{j}} L_{X}(j, k)^{q} \sim K_{q} 2^{j \zeta(q)}, 2^{j} \rightarrow 0 .
$$

Eq. 1 serves for the estimation of the so-called scaling exponents $\zeta(q)$ by linear regressions [17]. In turn, the function $\zeta(q)$ can be related to $\mathcal{D}(h)$ via a Legendre transform:

$$
\mathcal{L}(h)=\inf _{q}(1+q h-\zeta(q))
$$

which provides an upper bound estimate, $\mathcal{L}(h) \geq \mathcal{D}(h)$ for large classes of processes [17].

\section{Multifractal analysis: Practice}

Prior to multifractal analysis, it is common to consider the sum of the squared wavelet coefficients, closely related to the Fourier spectrum and covariance function of $X$ [1], [2]. For processes with scale free and/or multifractal dynamics, this sum, $S_{2}(j)$, behaves, over a wide range of scales, as [1]:

$$
S_{2}(j)=\frac{1}{n_{j}} \sum_{k=1}^{n_{j}} d_{X}^{2}(j, k) \sim F_{2} 2^{j 2 H},
$$

where $H$ is often referred to as the Hurst or self-similarity or long-memory exponent of $X$, and can be related to the second-order multifractal scaling exponent as $2 H=\zeta(2)$.

For most well-behaved multifractal processes, the scaling exponents can be approximated by truncating the polynomial expansion, $\zeta(q)=\sum_{p>1} c_{p} q^{p} / q$ !, whose coefficients $c_{p}$ can be theoretically related to the cumulants of order $p$ of $C_{p}(j)$ of the variables $\log _{2} L_{X}(j, k)$ as [17]:

$$
C_{p}(j)=c_{p}^{0}+c_{p} j .
$$

Function $C_{1}(j)$ conveys information mostly driven by the covariance function of the increments $Y$ of process $X$ and is hence very much related to the function $\log _{2} S_{2}(j)$ [17]. Along the same line, $2 H=\zeta(2)$ implies that $H=c_{1}+c_{2}+$ $2 c_{3} / 2+3 c_{4} / 4+\ldots$ For most multifractal processes, $\forall p \geq 2$, $c_{1} \gg c_{p}$, and hence that $H \simeq c_{1}$. Conversely, functions $C_{p}(j)$ for $p \geq 2$ convey information on temporal dynamics 
beyond second order statistics, i.e., not already encoded in the covariance function. When the $c_{p} \neq 0$, for some $p \geq 2$, such information is referred to as the multifractality of $X$. Moreover, Eq. 4 serves for the estimation of $c_{p}$ by linear regressions [17]. In turns, it has been shown that $\mathcal{D}(h)$ can be efficiently approximated as [2]:

$$
\mathcal{D}(h)=1+\frac{c_{2}}{2}\left(\frac{h-c_{1}}{c_{2}}\right)^{2}-\frac{c_{3}}{6}\left(\frac{h-c_{1}}{c_{2}}\right)^{3}+\ldots
$$

Descriptors $c_{1}, c_{2}, c_{3}, c_{4}, \ldots$, corresponding respectively to location of the maximum, width, asymmetry, flatness, ..., of $\mathcal{D}(h)$ thus summarize the temporal dynamics of $X$.

\section{Scale-free Models}

Fractional Brownian motion (fBm). Defined as the only Gaussian self-similar process with stationary increments, $\mathrm{fBm}$, $B_{H}(t)$, has its entire statistics controlled by the unique parameter $0<H<1$ [13]. This thus implies that $\zeta(q)=q H$ and $D(h)=\delta(h-H)$.

Fractional Brownian motion in multifractal time (MFfBm). Introduced by Mandelbrot for a better modeling of asset price fluctuations [12], MF-fBm is defined by composing an $\mathrm{fBm} B_{H_{0}}$ with a multifractal measure $A, \mathcal{B}_{H_{0}, A}(t) \triangleq$ $B_{H_{0}}(A(t))$. For such processes, $\zeta(q), D(h)$ and shape parameters $c_{1}, c_{2}, c_{3}, c_{4}, \ldots$ depend jointly on $H_{0}$ and on parameters entering the definition of $A$ [7], [17]. The Hurst parameter associated with $\mathcal{B}_{H_{0}, A}$ can be defined as $H=\zeta(2) / 2$.

\section{E. Shuffling procedures}

To understand what aspects of temporal dynamics are actually encoded in multifractal properties, the estimated multifractal properties of original data $X$, summarized by $H, c_{1}, c_{2}, c_{3}, c_{4}$, will be compared to those of surrogate copies, obtained by applying several different shuffling procedures to the increment process $Y$ of $X, Y(t)=X(t+1)-X(t)$.

Procedure Sign-Shuffling consists in creating a surrogate process $Y^{(S)}$ by rewriting $Y(t)=\operatorname{sgn}(Y(t)) \times|Y(t)|$ and randomly permuting (or shuffling) $\operatorname{sgn}(Y(t))$ while preserving unaltered moduli: $Y^{(S)}(t) \triangleq \operatorname{shuffle}(\operatorname{sgn}(Y(t)) \times|Y(t)|$.

Procedure Modulus-Shuffling consists in creating a surrogate process $Y^{(M)}$ by shuffling variables $\left.\mid Y(t)\right) \mid$ while preserving unaltered signs: $Y^{(M)}(t) \triangleq \operatorname{sgn}(Y(t) \times$ shuffle $(|Y(t)|)$.

Procedure Full-Shuffling consists in creating a surrogate process $Y^{(F)}$ by shuffling both signs and moduli: $Y^{(F)}(t) \triangleq$ $\operatorname{shuffle}(\operatorname{sgn}(Y(t)) \times \operatorname{shuffle}(|Y(t)|)$.

Surrogate processes $X^{(S)}, X^{(M)}$ and $X^{(F)}$ are constructed as the primitives of $Y^{(S)}, Y^{(M)}$ and $Y^{(F)}$, respectively.

\section{SHUFFLING SYNTHETIC MULTIFRACTAL PROCESSES}

\section{A. Monte Carlo simulations set-up}

The three shuffling procedures described above are applied to 1000 independent copies of MF-fBm, defined from $\mathrm{fBm}$ and Compound Poisson cascades, used as multifractal measures [7]. Long time series of $2^{18}$ samples are used, to obtain statistically relevant results hence permitting a discussion not confused by estimation performance issues. Multifractal
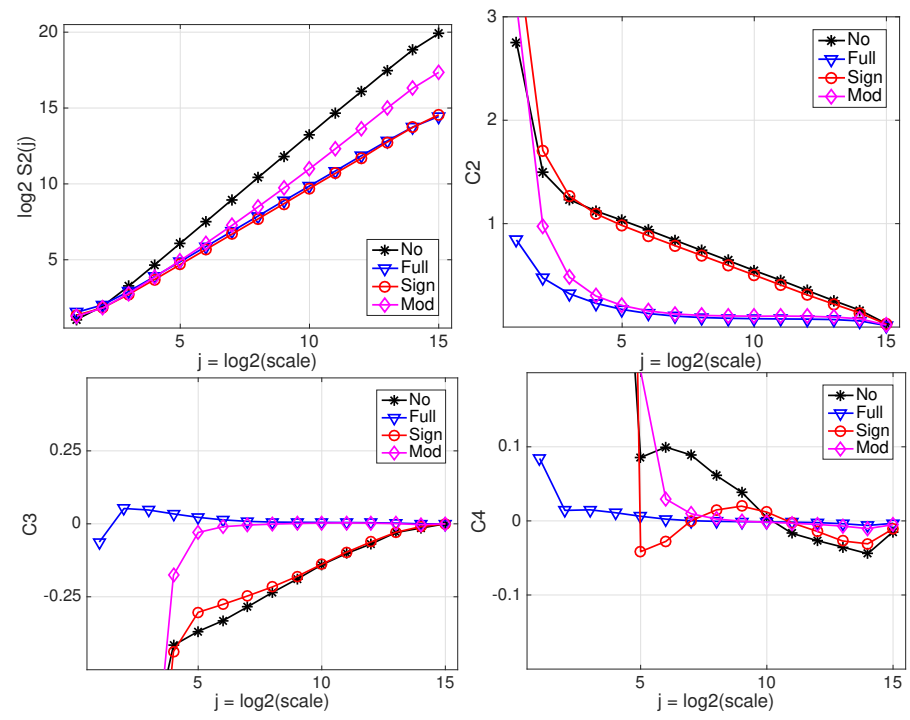

Fig. 1. Self-similarity, multifractality and shuffling. Functions $\log _{2} S_{2}(j)$ (top left), $C_{2}(j)$ (top right), $C_{3}(j)$ (bottom left) and $C_{4}(j)$ (bottom right) estimated from MF-fBm compared to those obtained on shuffled surrogates. Original data $X$ (black stars), Sign-Shuffled $X^{(S)}$ (red circles), ModulusShuffled $X^{(S)}$ (pink diamonds), Full-Shuffled $X^{(F)}$ (blue triangles). Averages over 1000 independent copies.

TABLE I

ESTIMATED MULTIFRACTAL PARAMETERS (AND CONFIDENCE INTERVALS) FOR MF-FBM COMPARED TO THOSE OBTAINED FROM SHUFFLED SURROGATES. AVERAGES OVER 1000 INDEPENDENT COPIES.

\begin{tabular}{|c||c|c|c|c|}
\hline & $H$ & $c_{2}$ & $c_{3}$ & $c_{4}$ \\
\hline \hline Data & $0.70(0.01)$ & $-0.140(0.002)$ & $0.054(0.003)$ & $-0.026(0.006)$ \\
F & $0.49(0.01)$ & $-0.007(0.001)$ & $-0.001(0.002)$ & $-0.001(0.001)$ \\
S & $0.50(0.01)$ & $-0.135(0.002)$ & $0.052(0.003)$ & $-0.013(0.004)$ \\
M & $0.66(0.01)$ & $-0.006(0.001)$ & $-0.000(0.002)$ & $-0.003(0.001)$ \\
\hline
\end{tabular}

analysis is conducted using least asymmetric orthonormal wavelets with $N_{\psi}=3$ vanishing moments. Other choices yield equivalent results. Synthesis and analysis procedures were devised by ourselves and will be made publicly available ${ }^{1}$. Estimation results, obtained as averages across independent copies, are reported for one set of multifractal attributes (cf. Table I), equivalent results are yet obtained with other sets of multifractal attributes. Because function $C_{1}(j)$ conveys an information almost equivalent to that in $\log _{2} S_{2}(j)$, it it not plotted in Fig. 1 (nor later in Figs. 2 and 3) for space reasons. Also, estimates of $c_{1}$, close to those of $H$, are not reported in Table I (nor later in Table II).

\section{B. Self-similarity, multifractality and shuffling}

Shuffling and scale invariance. Fig. 1 shows that estimated functions $C_{p}(j)$ and $\log _{2} S_{2}(j)$ for original data essentially consist of straight lines as functions of octave $j$, as expected from Eqs. 3-4 for exactly scale-free synthetic processes. Deviations from straight lines observed at the finest scales, even for exactly scale-free and multifractal processes, are due to a bias

\footnotetext{
${ }^{1}$ http://www.ens-lyon.fr/PHYSIQUE/Equipe3/MultiFracs/
} 
induced by wavelet leaders: By definition, the computation of wavelet leaders requires wavelet coefficients at finer scales, which are not available because of discretization. This has been documented elsewhere [17] and is no longer discussed here. Importantly, Fig. 1 shows that functions $\log _{2} S_{2}(j)$ and $C_{p}(j)$ consist of straight lines as in Eqs. 3-4 also for shuffled data, thus indicating that scale-free dynamics still exist in all shuffled time series, and raising the question of whether LRD or multifractal properties were modified or not.

Shuffling, multifractality and LRD. Comparing now the functions $C_{p}(j)$, for $p \geq 2$, in Fig. 1, clearly shows that i) they are not altered by Sign-Shuffling, and that ii) they are systematically altered by Modulus-Shuffling and FullShuffling and transformed into functions that no longer depend on $j$. This is confirmed in Table I that shows that $c_{p}$, for $p \geq 2$ of $X^{(S)}$ are essentially similar to those of $X$, while those of $X^{(M)}$ and $X^{(F)}$ are close to 0 . These empirical facts clearly show that multifractality is destroyed by shuffling moduli of the increments of $X$ but not by shuffling signs.

Moreover, inspection of the functions $\log _{2} S_{2}(j)$ in Fig. 1 shows that all forms of shuffling affect the second order statistics of the process (the correlation structure). However, a careful examination of the plots shows that in the limit of coarse scales $2^{j} \rightarrow+\infty$, the impact of Modulus-Shuffling vanishes. This is confirmed by Table I, reporting estimates obtained for coarse scales and showing that estimated $H$ for $X^{(M)}$ are much closer to those of $X$ than the estimates from $X^{(S)}$ which are closer to 0.5 .

This leads to the following conclusions. First, SignShuffling impacts LRD for multifractal processes, a result highly reminiscent of that proving that Sign-Shuffling impacts LRD for self-similar processes (cf. [13]). Second, ModulusShuffling impacts multifractality in an intricate way: the impacts concentrates mostly at fine scales and vanishes in the asymptotic limit of coarse scales.

\section{Multifractality IN FinANCE}

\section{A. Dataset and analysis}

The dataset analyzed here consists of a collection of $p=$ 211 daily asset price time series chosen within the basket defining the Eurostoxx600 index, recorded over 12 years, from December, the 14th, 2001 to January, the 24th, 2013, for a total sample size of $n=2900$. This selection constitutes a relevant basket that could potentially be used in practice by investment firms. An example of such time series is provided in Fig. 2.

Multifractal analysis is applied to these time series and to the surrogate time series obtained by the shuffling procedures, with the same parameter setting as that described for Monte Carlo simulations in Section III-A above.

\section{B. Results and discussion}

For illustration and pedagogy, Fig. 2 displays, for an arbitrarily chosen asset, the price time series and compares the functions $\log _{2} S_{2}(j)$ and $C_{p}(j)$ and Legendre spectra $\mathcal{L}(h)$ for the original data and shuffled surrogates. It shows that the functions $C_{p}(j)$ for $p \geq 2$ obtained after Sign-Shuffling
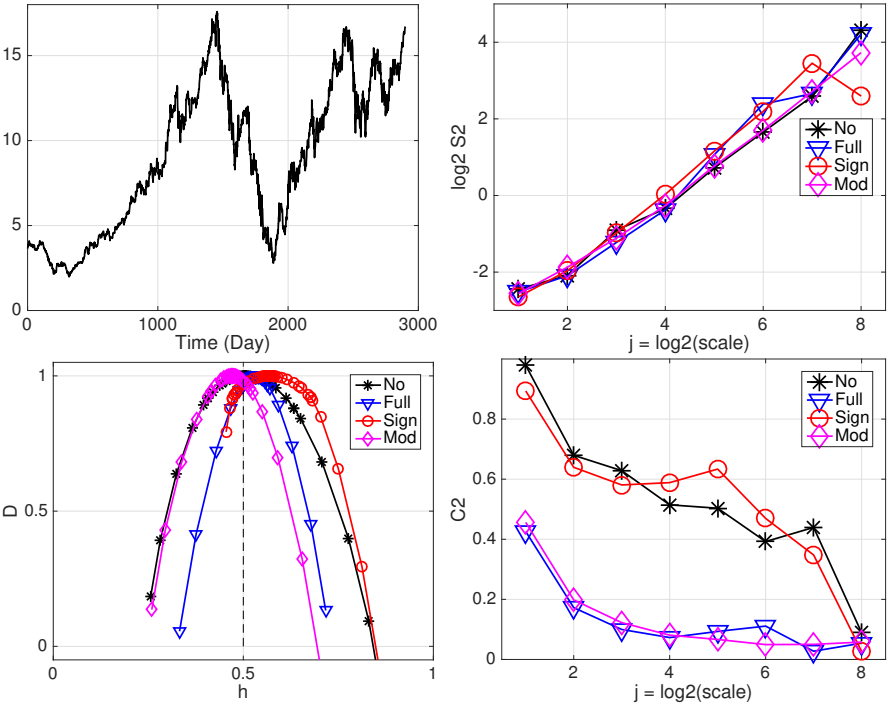

Fig. 2. Self-similarity and multifractality on one asset price. Time series (top left), $\log _{2} S_{2}(j)$ (top right), estimated Legendre spectrum $\mathcal{L}(h)$ (bottom left), $C 2(j)$ (bottom right). Original data $X$ (black stars),Sign-Shuffled $X^{(S)}$ (red circles), Modulus-Shuffled $X^{(S)}$ (pink diamonds), Full-Shuffled $X^{(F)}$ (blue triangles).
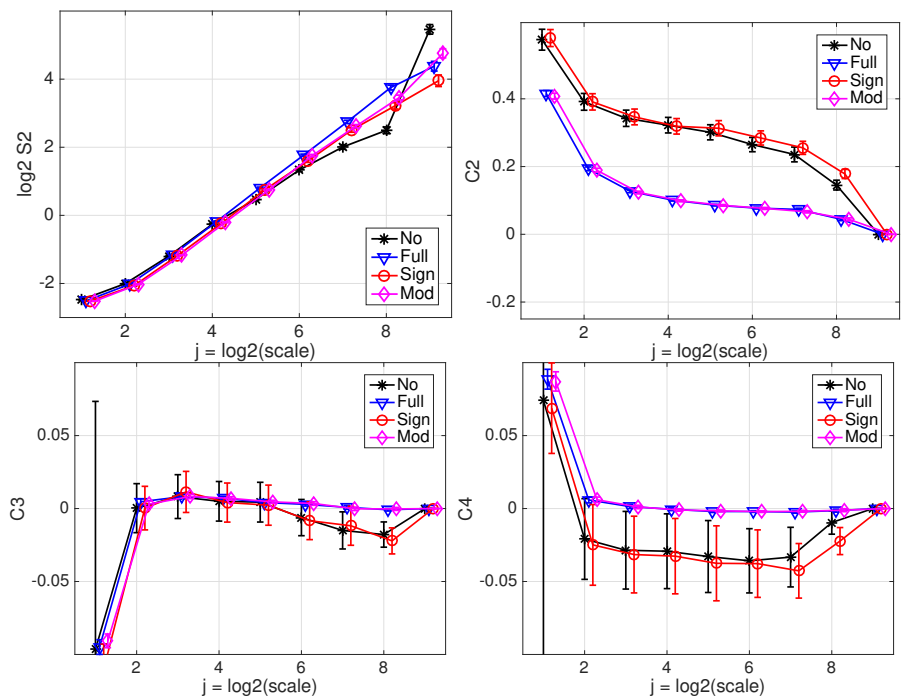

Fig. 3. Self-similarity and multifractality in Eurostoxx basket. Functions $\log _{2} S_{2}(j)$ (top left), $C_{2}(j)$ (top right), $C_{3}(j)$ (bottom left) and $C_{4}(j)$ (bottom right) averaged over 211 Eurostoxx asset price time series and compared to those obtained as averages on shuffled surrogates. Original data $X$ (black stars),Sign-Shuffled $X^{(S)}$ (red circles), Modulus-Shuffled $X^{(S)}$ (pink diamonds), Full-Shuffled $X^{(F)}$ (blue triangles).

very well match those of the original data, while the functions $C_{p}(j)$ for $p \geq 2$ obtained after Modulus-Shuffling are strongly modified and no longer depend on scales $2^{j}$, when the biased short time scales are disregarded. On this example, one sees that Modulus-Shuffling suppresses multifractality while SignShuffling does not. Fig. 2 also clearly shows that functions $\log _{2} S_{2}(j)$ and $C_{p}(j)$ are not altered by any form of shuffling: this is because the original time series show no correlation structure, shuffling can hence not destroy it. 
TABLE II

ESTIMATED MULTIFRACTAL PARAMETERS (AND CONFIDENCE INTERVALS) FOR EUROSTOXX ASSET PRICE TIMES SERIES COMPARED TO THOSE OBTAINED FROM SHUFFLED SURROGATES

\begin{tabular}{|c||c|c|c|c|}
\hline & $H$ & $c_{2}$ & $100 \times c_{3}$ & $10 \times c_{4}$ \\
\hline \hline Data & $0.47(0.01)$ & $-0.095(0.008)$ & $-0.26(0.96)$ & $0.298(0.144)$ \\
F & $0.46(0.01)$ & $-0.029(0.002)$ & $-0.33(0.06)$ & $-0.018(0.007)$ \\
S & $0.43(0.01)$ & $-0.092(0.008)$ & $-0.47(0.82)$ & $0.284(0.141)$ \\
M & $0.48(0.01)$ & $-0.029(0.002)$ & $-0.33(0.06)$ & $-0.018(0.007)$ \\
\hline
\end{tabular}

To draw conclusion at the entire basket level, averages (and confidence intervals) for functions $\log _{2} S_{2}(j)$ and $C_{p}(j)$ are computed over assets (after a suitable normalization of increment powers, an operation that does not alter time dynamics), as if they were independent realizations of one same financial process. This approach is common in econophysics and relies on the existence of universal behaviors in economic and financial systems [15]. Fig. 3 compares basket averaged functions $\log _{2} S_{2}(j)$ and $C_{p}(j), p=2,3,4$ for the original data and for shuffled surrogates.

Fig. 3 clearly shows that functions $C_{p}(j)$ for $p \geq 2$ obtained after Sign-Shuffling almost perfectly superimpose with those of the original data. Conversely, it also shows that functions $C_{p}(j)$ for $p \geq 2$ obtained after Modulus-Shuffling are strongly altered and are close to constant with respect to scales $2^{j}$, when the biased short time scales are disregarded. This is further confirmed in Table II that reports estimated multifractal parameters $c_{2}, c_{3}$ and $c_{4}$ from the averaged $C_{p}(j), p=2,3,4$ for original and shuffled data. It can be seen that estimates of Sign-Shuffled surrogates and original data are in close agreements while estimates of Modulus-Shuffled surrogates strongly differ and are close to 0 . Therefore, Sign-Shuffling leaves multifractility unaltered while Modulus-Shuffling quasitotally destroys it, and, remarkably, at any statistical order.

Fig. 3 also shows that functions $\log _{2} S_{2}(j)$ are essentially not altered by any form of shuffling. Further, Table II confirms that estimated $H$ are essentially identical for original and shuffled data. In addition, these estimated $H$ are found close to 0.5 thus confirming the absence of long memory and even the absence of any autocorrelation in asset price fluctuations, which can hence not be altered by shuffling. Incidentally, this also validates that shuffling per se does not create any spurious form of long memory (nor of multifractality).

Table II also shows that the asset price times series considered here display a significant multifractality (large $c_{2}$ ) that tends to involves only even cumulants $\left(c_{3} \simeq 0\right.$ while $c_{4}$ significantly departs from 0 ). Interestingly, this indicates that multifractality does not account for any asymmetry in such data, reinforcing the relation to modulus, hence to volatility.

\section{CONCLUSIONS AND PERSPECTIVES}

The present work explored the relations between multifractality and the time dynamics of signs and modulus in synthetic and financial time series. Using the state-of-the-art wavelet leader based multifractal formalism, and conducting Monte Carlo simulations over synthetic copies of reference multi- fractal processes (fractional Brownian motion in multifractal time), and by comparing estimated multifractal properties of original and shuffled surrogates, we have established a clear and generic relation between multifractality and the temporal dynamics of the increment moduli. Conversely, we have shown that mutlifractality is not quantifying the temporal dynamics of increment signs. It has also been shown that the temporal dynamics of increment signs is coded in long memory, while the possible impact of the temporal dynamics of increment moduli on long memory concentrates on short time scales and asymptotically vanishes at coarse scales.

For asset price time series, the achieved results unambiguously indicate that multifractality is not related to the temporal dynamics of the returns but is clearly tied to the temporal dynamics of volatility. These findings are of interest for financial engineering at large, e.g., suggesting that multifractality can not be used for return prediction, but could be involved in volatility forecasting.

\section{REFERENCES}

[1] P. Abry, R. Baraniuk, P. Flandrin, R. Riedi, and D. Veitch. Multiscale network traffic analysis, modeling, and inference using wavelets, multifractals, and cascades. IEEE Signal Processing Magazine, 3(19):28-46, May 2002.

[2] P. Abry, S. Jaffard, and H. Wendt. Irregularities and scaling in signal and image processing: Multifractal analysis. In Benoit Mandelbrot: A Life in Many Dimensions, pages 31-116. World Scientific, 2015.

[3] A. Arneodo, J-F Muzy, and D. Sornette. Direct causal cascade in the stock market. The European Physical Journal B-Condensed Matter and Complex Systems, 2(2):277-282, 1998.

[4] E. Bacry, A. Kozhemyak, and J.-F. Muzy. Continuous cascade models for asset returns. Journal of Economic Dynamics and Control, 32(1):156-199, 2008.

[5] J-P Bouchaud, Marc Potters, and Martin Meyer. Apparent multifractality in financial time series. The European Physical Journal B-Condensed Matter and Complex Systems, 13(3):595-599, 2000.

[6] L. Calvet, A. Fisher, and B. Mandelbrot. The multifractal model of asset returns. Cowles Foundation Discussion Papers: 1164, 1997.

[7] P. Chainais, R. Riedi, and P. Abry. On non-scale-invariant infinitely divisible cascades. IEEE transactions on Information Theory, 51(3):10631083, 2005.

[8] T. Di Matteo, T. Aste, and M. M Dacorogna. Long-term memories of developed and emerging markets: Using the scaling analysis to characterize their stage of development. Journal of Banking \& Finance, 29(4):827-851, 2005.

[9] T. Lux. The markov-switching multifractal model of asset returns: Gmm estimation and linear forecasting of volatility. Journal of business \& economic statistics, 26(2):194-210, 2008.

[10] T Lux and M Segnon. Multifractal models in finance: Their origin, properties and applications, 2016.

[11] S. Mallat. A Wavelet Tour of Signal Processing. Academic Press, San Diego, CA, 1998.

[12] B. B. Mandelbrot. A multifractal walk down Wall Street. Sci. Am., 280(2):70-73, 1999.

[13] V. Pipiras and M. S Taqqu. Long-range dependence and self-similarity, volume 45. Cambridge university press, 2017.

[14] D. Sornette, Y. Malevergne, and J.-F. Muzy. Volatility fingerprints of large shocks: endogenous versus exogenous. In The Application of Econophysics, pages 91-102. Springer, 2004.

[15] H. E. Stanley, L. A. N. Amaral, P. Gopikrishnan, and V. Plerou. Scale invariance and universality of economic fluctuations. Physica A: Statistical Mechanics and its Applications, 283(1-2):31-41, 2000.

[16] A. K. Tiwari, C. T. Albulescu, and S.-M. Yoon. A multifractal detrended fluctuation analysis of financial market efficiency: Comparison using dow jones sector etf indices. Physica A: Statistical Mechanics and its Applications, 483:182-192, 2017.

[17] H. Wendt, P. Abry, and S. Jaffard. Bootstrap for empirical multifractal analysis. IEEE Signal Proc. Mag., 24(4):38-48, 2007. 